\title{
Advancing research in the exciting field of emergency medicine
}

\author{
Shu-Ling Chong ${ }^{1}$, MRCPCH, MPH, Marcus Eng Hock Ong ${ }^{2,3}$, MMed, MPH
}

W hat started out in 1948 as a casualty department in the Singapore General Hospital ${ }^{(1)}$ has since evolved into an islandwide network of eight adult and two paediatric emergency departments (EDs). EDs in our restructured hospitals cater to the needs of an estimated 2,500 adult patients daily. ${ }^{(2)}$ With such large daily patient loads, EDs form a natural resource pool to study diagnostic dilemmas and specific disease states. Emergency medicine is increasing in complexity and sophistication, with subspecialties in emergency cardiac care, emergency trauma care, prehospital emergencies, injury control and prevention, paediatric emergency medicine, emergency toxicology, emergency imaging, geriatric emergency medicine, observation medicine, emergency airway, and disaster medicine. In this issue of the Singapore Medical Journal, we get a glimpse of the breadth and reach of both primary care and ED research in addressing the needs of our population.

With the silver tsunami and increasing demand for tertiary medical care, our EDs are currently burdened with overcrowding and long waiting times. Using established administrative criteria, Oh et $\mathrm{al}^{(3)}$ identified patient subgroups that account for inappropriate attendances in the ED. While stratifying patients by the acuity of their needs is important, understanding the level of knowledge and confidence of primary care doctors in managing various medical urgencies is also crucial in the delicate balance to right-site patients. Meanwhile, $\mathrm{Ng}$ et $\mathrm{al}^{\left({ }^{(4)}\right.}$ highlighted a lack of confidence among primary care doctors in Kuala Lumpur when managing in-flight emergencies, suggesting that building primary physicians' confidence and improving their management of common medical conditions would address some of the gaps we currently face. By reducing inappropriate attendances and allowing ED physicians to focus on the sickest patients, Ibrahim et $\mathrm{al}^{(5)}$ demonstrated in their work on acute aortic dissection that we can better target our resources so as to minimise the occurrence of missed diagnoses among critically ill patients.

ED physicians are increasingly cognisant of the importance of injury surveillance and research that can fuel upstream interventions. Liew et $\mathrm{al}^{(6)}$ demonstrated that electric scooter injuries are on the rise and consuming an increasing amount of healthcare resources, while Cha et $\mathrm{al}^{(7)}$ studied injuries from all types of personal mobility devices, highlighting that electric bicycle injuries tend to be more severe. Looking specifically at road safety for children, Tan et al ${ }^{(8)}$ performed a qualitative study among parents to understand non-compliance behaviours towards child car restraints in Singapore, highlighting actionable points such as the importance of legal enforcement on the roads and improving installation services for child car restraints. These efforts can be meaningfully harnessed if such data is conveyed to the relevant agencies for policymakers to effect change.

Prehospital care in Singapore has evolved to meet the challenges of a small city-state and its dense population. ${ }^{(9)}$ Outcomes for out-of-hospital cardiac arrest are intricately linked to early cardiopulmonary resuscitation (CPR) and early defibrillation. ${ }^{(10)}$ In order to improve the local bystander defibrillation rate, Chua et $\mathrm{al}^{(11)}$ highlighted the importance of an automated external defibrillator (AED) registry. Using a publicly available smartphone app (myResponder), volunteers can be alerted to nearby cases of cardiac arrest and the geolocation of reported AEDs. Increased uptake and continued use of the myResponder app will increase local bystander CPR and defibrillation rates.

Emergency medicine research is challenging, not least because of the breadth of illnesses and injuries that ED physicians are expected to master. The topics covered in this issue of the journal are but the tip of the iceberg in current emergency medicine research priorities. ${ }^{(12)}$ Gains from the heavy clinical service load in all EDs include the large amounts of data on patients of all ages with a variety of undifferentiated complaints. In order to better power ED trials and ensure generalisability, both adult and paediatric EDs in Singapore are currently participating in and leading multicentre ED studies in Asia and the world. These span prehospital emergency care, cardiac arrest research, and epidemiology studies on paediatric emergencies and traumatic brain injuries.

Being the portal of entry to each of our tertiary hospitals and institutions, EDs represent the capability and tenacity of modern day medicine. As such, patients coming to our EDs deserve the best care we can deliver. As emergency physicians, we believe that quality emergency medical care should be "evidence-based, integrated, adaptable and efficient" ${ }^{(13)}$ Integrated care in the practice of emergency medicine must be fuelled by strong collaboration with other specialties and disciplines. Our dream of delivering quality care can only be realised within a culture that treasures and supports ED research as part of the greater mission for patients' good.

\section{REFERENCES}

1. Lim SH, Anantharaman V. Emergency medicine in Singapore: past, present, and future. Ann Emerg Med 1999; 33:338-43.

2. Ministry of Health, Singapore. Attendances at Emergency Medicine Departments [online]. Available at: https://www.moh.gov.sg/resources-statistics/healthcare-

${ }^{1}$ Department of Emergency Medicine, KK Women's and Children's Hospital, ${ }^{2}$ Department of Emergency Medicine, Singapore General Hospital, ${ }^{3} \mathrm{Health}$ Services and Systems Research, Duke-NUS Medical School, Singapore

Correspondence: Dr Chong Shu-Ling, Senior Staff Physician, Department of Emergency Medicine, KK Women's and Children's Hospital, 100 Bukit Timah Road, Singapore 299899. Chong.Shu-Ling@kkh.com.sg 
institution-statistics/attendances-at-emergency-medicine-departments. Accessed August 16, 2019.

3. Oh HC, Chow WL, Gao Y, et al. Factors associated with inappropriate attendances at the emergency department of a tertiary hospital in Singapore. Singapore Med J 2020; 61:75-80.

4. Ng WL, Abdullah N. Knowledge, confidence and attitude of primary care doctors in managing in-flight medical emergencies: a cross-sectional survey. Singapore Med J 2020; 61:81-85.

5. Ibrahim I, Chua MT, Tan DW, et al. Impact of 24-hour specialist coverage and an on-site CT scanner on the timely diagnosis of acute aortic dissection. Singapore Med J 2020; 61:86-91.

6. Liew YK, Wee CPJ, Pek JH. New peril on our roads: a retrospective study of electric scooter-related injuries. Singapore Med J 2020; 61:92-95.

7. Cha Sow King C, Liu M, Patel S, et al. Injury patterns associated with personal mobility devices and electric bicycles: an analysis from an acute general hospital in Singapore. Singapore Med J 2020; 61:96-101.
8. Tan RMR, Dong C, Shen GQ, et al. Parental knowledge and beliefs on the use of child car restraints in Singapore: a qualitative study. Singapore Med J 2020; 61:102-107.

9. Ho AF, Chew D, Wong TH, et al. Prehospital trauma care in Singapore. Prehosp Emerg Care 2015; 19:409-15.

10. Hallstrom A. Public-access defibrillation and survival after out-of-hospital cardiac arrest. N Engl J Med 2004; 351:637-46

11. Chua SYI, Ng YY, Ong MEH. Getting R-AEDI to save lives in Singapore. Singapore Med J 2020; 61:60-62.

12. Smith J, Keating L, Flowerdew L, et al; JLA EM PSP Steering Group. An emergency medicine research priority setting partnership to establish the top 10 research priorities in emergency medicine. Emerg Med J 2017; 34:454-6.

13. State of Emergency Medical Care in Singapore Workgroup. State of emergency medical care in Singapore: addressing challenges and charting directions. Singapore: College of Emergency Physicians, Academy of Medicine, Singapore and Society for Emergency Medicine in Singapore, 2015. 